\title{
Psychogenic Elaboration of Simple Partial Seizures
}

\author{
Jaideep Kapur, Ashok Pillai, and Thomas R. Henry \\ Department of Neurology, University of Michigan, Ann Arbor, Michigan, U.S.A.
}

Summary: Seizures that cause loss of consciousness (LOC) can be classified as epileptic or nonepileptic based on evaluation of ictal semiology and analysis of changes in EEG events, recorded with continuous scalp EEG and video monitoring. We report 3 patients who had hippocampal electrographic seizures documented with intracranial EEG recording with no accompanying scalp EEG change immediately preceding psychogenic unresponsiveness. Each patient also had complex partial seizures (CPS) originating in the hippocampus. Some individuals can have complex interactions of epileptic and nonepileptic seizures. Key Words: Temporal lobe epilepsyNonepileptic seizure-Pseudoseizure-Complex partial seizure.
Closed-circuit television EEG (CCTV-EEG) monitoring with scalp electrodes has been used extensively to distinguish epileptic and nonepileptic seizures (1-10). Unchanged, adequately recorded scalp EEG during transient unconsciousness supports a diagnosis of nonepileptic seizure in the absence of evidence for frontal lobe seizures or severe widespread interictal cerebral dysfunction.

Gumnit and Gates (11) hypothesized that a partial seizure which remains confined to a small part of the brain and has no scalp EEG representation may trigger a nonepileptic seizure. These events should be detectable with intracranial EEG recording, but no examples of this hypothesized phenomenon have been documented with simultaneous intracranial and scalp EEG and video recording. We now report 3 patients who demonstrated this phenomenon during intracranial monitoring.

\section{CASE REPORTS}

We reviewed 50 consecutive intracranial EEG monitoring procedures performed in patients with medically refractory complex partial seizures (CPS) to detect events of unresponsiveness not associated with simultaneous electrographic seizure activity. Three patients had electrographic seizures re-

Received March 17, 1994; revision accepted March 24, 1995. Address correspondence and reprint requests to Dr. J. Kapur at Department of Neurology, Neuroscience Building, 1103 E. Huron St., Ann Arbor, MI 48104-1687, U.S.A. stricted to one hippocampus and the adjacent temporal pole without accompanying changes on scalp EEG that preceded unresponsiveness.

The patients were evaluated according to the University of Michigan epilepsy surgery protocols (12). Depth electrodes with eight contacts $(1-\mathrm{cm}$ spacing between contacts) were placed stereotaxally under magnetic resonance imaging guidance in mesial temporal structures bilaterally by a posteroanterior approach (passing through the hippocampus to the amygdala) to record intracranial EEG. Patients 2 and 3 also had six-contact depth electrodes placed in mesial frontal structures by a superoinferior approach (passing through the anterior cingulate gyrus to the gyrus rectus). In addition, six subdural electrode strips, each with four contacts, were placed bilaterally and symmetrically, one each over inferotemporal, anterolaterotemporal and dorsolaterofrontal regions. Scalp EEG was recorded from electrodes placed according to the International 10-20 system in each patient.

Interictal scalp EEG showed unilateral or bilateral temporal slowing and epileptiform discharges in each patient; there were no extratemporal abnormalities. Before intracranial monitoring, scalp EEG video-monitoring had demonstrated ictal EEG discharges during every seizure that involved unconsciousness. Each patient underwent anterior temporal lobectomy after monitoring. All 3 had hippocampal sclerosis on histopathologic examination and have remained free of CPS for $>1$ year postoperatively. 


\section{Case 1}

A 22-year-old woman whose epilepsy was caused by pneumococcal meningitis at age 7 months had seizure onset at age 15 years. CPS began with an epigastric sensation and an illusion of increased distance from her visual and auditory environment. Neurological examination was normal. Verbal I.Q. (VIQ) was 89 and performance I.Q. (PIQ) was 109 (Wechsler Adult Intelligence Scale-Revised). She had evidence of nonpsychotic depression on the clinical interview and the Minnesota Multiphasic Personality Inventory (MMPI).

After several days of intracranial monitoring, the patient began to express great anxiety over the localization studies and her potential for resective surgery. She reported concentrating hard on each aura in hopes of obtaining diagnostically useful seizure activity. On the sixth day of monitoring, the patient had a right hippocampal electrographic seizure lasting $99 \mathrm{~s}$. There was no change in EEG activity at other intracranial or scalp electrodes. Three seconds after the onset of the electrographic seizure, the patient reported an aura and then became motionless with eyes open. After the ictal electrographic discharge ended, the patient did not respond to verbal and tactile stimuli for $5 \mathrm{~min} 26 \mathrm{~s}$. During this episode, a neurologist noted flaccidity, no abnormal movements, and no response to noxious stimuli. After resolution of the electrographic and behavioral changes, the patient could not recall any events during this spell. Her parents confirmed that the behavior during this event did not resemble that of her habitual seizures.
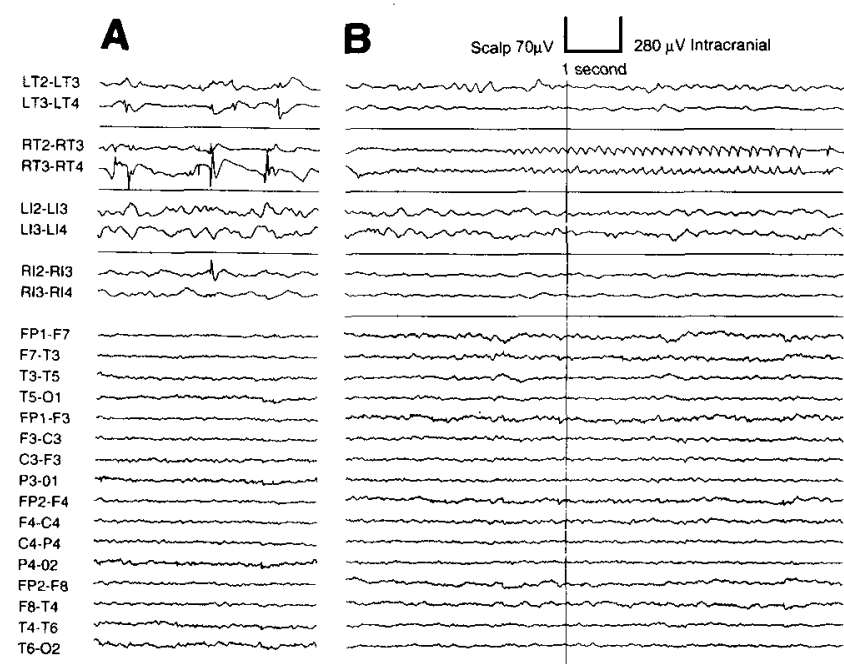

She had six CPS captured with intracranial electrodes. These seizures began with her typical aura and exclusively right hippocampal electrographic seizure activity, followed by $\leqslant 60 \mathrm{~s}$ altered consciousness and repetitive masticatory and hand movements for which she subsequently was amnesic. These CPS accompanied electrographic seizure activity involving multiple sites bilaterally.

She had 37 electrographic seizures confined to the right hippocampus which were either subclinical or were reported only as auras. Behavior during several of these restricted hippocampal seizures was tested by a neurologist or other trained personnel. None of the unpropagated hippocampal seizures was associated with altered consciousness or change in behavior except for a report of an aura.

\section{Case 2}

In a 21-year-old woman, CPS had onset at age 11 months after multiple febrile convulsions. CPS began with an aura of "difficult-to-describe" dizziness. Neurological examination was normal. VIQ was 88 and PIQ was 74 . She was anxious and mildly depressed. Results of the MMPI were normal except for evidence of an unusually high energy level and mild social isolation.

Despite discontinuation of antiepileptic drugs, the patient did not have seizures for many days after intracranial electrode implantation; she became deeply discouraged and expressed fears that epilepsy surgery would never occur. On the thirteenth day of monitoring, the patient stopped eating and leaned back as an electrographic seizure evolved
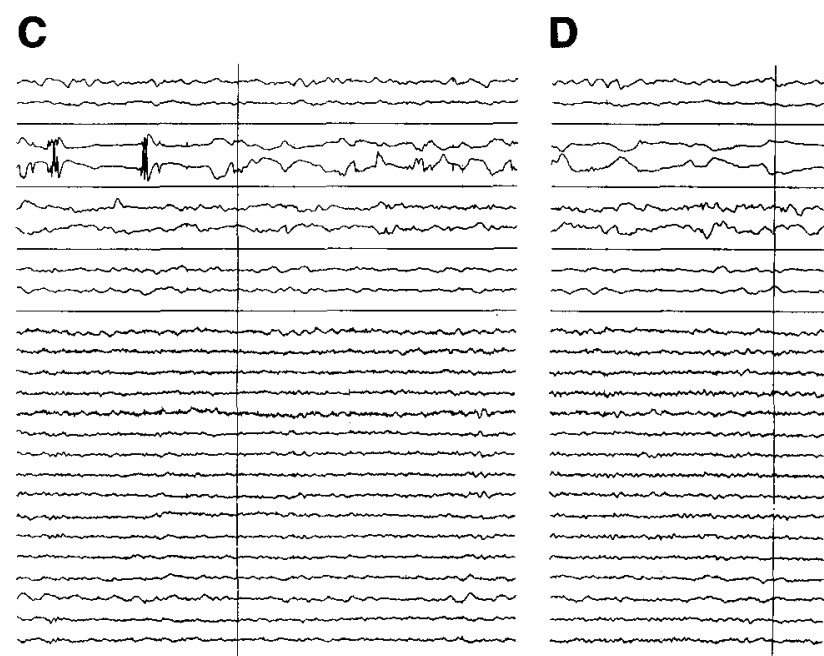

FIG. 1. Electroencephalographic recording during and after a right hippocampal electrographic seizure and unresponsiveness in patient 1. LT, RT, LI, RI, left hippocampal, right hippocampal, left inferotemporal, and right inferotemporal electrodes, respectively. The lower 16 channels show simultaneous scalp-recorded electrographic activity from electrodes labeled according to the International 10-20 system. A: Interictal activity at $5 \mathrm{~s}$ before ictal onset. B: Onset of a right hippocampal electrographic seizure. C: End of the electrographic discharge. D: Sample showing interictal activities including alpha activity over posterior head region while the patient remains unresponsive. B and $C$ are separated by $90 \mathrm{~s}$, and $\mathrm{C}$ and $\mathrm{D}$ are separated by $120 \mathrm{~s}$. 
from the left hippocampus, lasting $30 \mathrm{~s}$ and not involving other recording sites. She remained motionless and unresponsive to her mother's questioning for $60 \mathrm{~s}$ after the electrographic seizure. While she was unresponsive, her mother gave her a phrase to remember. Later, when normal she was unable to recall or recognize the phrase, nor could she remember other events during the spell. Her mother indicated that this event did not resemble any of the seizures she had previously witnessed.

Intracranial monitoring recorded five CPS. Each began with her typical aura and was associated with an exclusively left hippocampal electrographic seizure discharge, followed by a brief period ( $\leqslant 50 \mathrm{~s}$ ) of unconsciousness and nonstereotyped automatisms involving all limbs. During these CPS, electrographic seizure activity was present bilaterally both in temporal depth and subdural electrodes as well as in frontal electrodes. After resolution of postictal EEG slowing and behavioral changes, she remained amnesic for events that occurred when ictal discharges spread bilaterally.

She also had 34 electrographic seizures confined to the left hippocampus without propagation to other electrodes which were either subclinical or were reported only as auras. These unpropagated hippocampal seizures, during several of which detailed testing was performed, were not associated with decreased responsiveness or behavioral change.

\section{Case 3}

A 34-year-old woman had onset of CPS at age 17 years, 2 years after an episode of hypotension caused by a drug overdose. CPS began with an aura of déjà-vu experience and micropsia. Neurological examination was normal. VIQ was 84 and PIQ was 85. MMPI results were normal. Psychosocial assessment showed poor adjustment to her epilepsy, chronic anxiety and irritability, and a remote history of suicidal depression.

The patient tolerated the discomfort of intracranial electrode placement poorly, with increasing expressions of hopelessness and anger. On the fifth day of monitoring she awakened from sleep $28 \mathrm{~s}$ after onset of a left hippocampal seizure and reported an aura. The seizure lasted another $20 \mathrm{~s}$ and remained confined to the left hippocampus and temporal pole, but the scalp EEG showed no changes. The patient was motionless and unresponsive to touch and questions for $40 \mathrm{~s}$ after the electrographic seizure ended. Her spouse asked her to remember a phrase during this period, but once she was responsive she could not recall or recognize this phrase.
Her spouse had not previously observed similar behaviors.

During intracranial monitoring she had eight CPS. Each began with a typical aura occurring with an exclusively right hippocampal electrographic seizure discharge, with unresponsiveness lasting $<1$ min, early nonsense speech and, later, repetitive masticatory movements. During unresponsiveness, the electrographic seizure activity involved temporal and frontal electrodes bilaterally. After resolution of postictal electrographic slowing and behavioral changes, she remained amnesic for events during bihemispheric electrographic discharges.

She also had 145 unilateral hippocampal electrographic seizures which did not propagate to other electrodes and were either subclinical or were reported only as auras. During many of the unpropagated seizures, her behavior was tested and was never altered.

\section{DISCUSSION}

We conclude that highly localized electrographic partial seizures can trigger an immediate nonepileptic alteration in behavior during which the patient is unresponsive. There were no changes from baseline in scalp EEG activity during the epileptic and nonepileptic portions of these events. These spells did not suggest syncope or any other nonpsychogenic cause of unconsciousness. Each event occurred in an individual predisposed to psychological dysfunction. Each occurred in the setting of emotional distress. We believe that these events of nonepileptic unresponsiveness in each case represent psychogenic elaboration of a simple partial seizure (SPS).

There are several reasons to believe that our patients' unpropagated focal hippocampal electrographic seizures did not in themselves cause LOC. There was no evidence of seizure propagation beyond the hippocampus and temporal pole unilaterally. Specifically, ipsilateral frontal lobes and contralateral frontal or temporal lobes were not involved. Furthermore, each patient had many electrographic seizures that remained localized to these structures, with only an aura as a clinical manifestation or with no clinical accompaniment of the electrographic seizure. Two of our patients later reported the occurrence of the habitual aura during the highly focal electrographic seizure that preceded the psychogenic unconsciousness. Gloor (13) suggested that "impairment" of consciousness during a seizure may represent a peculiar motivationalattentional state in which patients are so absorbed in an experiential hallucination that they are obliv- 
ious to stimuli unrelated to the momentarily prevailing focus of attention. Our patients did not report experiencing intense concentration on their auras during these events, however.

Gloor has suggested that LOC in temporal lobe seizures can occur during unilateral temporal discharges (14). He based his view on early observations made in patients with continuously recorded intracranial EEG but no video-monitoring. No details of cognitive testing were presented. Theoretically, unilateral hippocampal discharge may spread to the brainstem and cause LOC. Functional mappings of hippocampal seizures in experimental animals (15) show that substantia nigra was activated only during severe limbic seizures, with concurrent activation of hippocampus bilaterally and thalamus. When hippocampal seizures were unilateral, there was no activation of substantia nigra. Unfortunately, replication of these studies in humans would require electrodes implanted in sites for which there is no current clinical indication. In a review, Gloor (13) called for detailed observations, similar to those we describe, to investigate the relationship between partial seizures and consciousness in humans.

In our 3 patients, scalp EEG remained unchanged during episodes of unconsciousness. Some CPS of frontal origin reportedly have no scalp EEG representation $(16,17)$ but, for several reasons, we doubt that any of our patients had such seizures. First, 2 of our patients had concurrent intracerebral recording of frontal lobe activity which showed no ictal discharge. Second, the semiology of the events did not suggest mesial frontal or orbitofrontal onset seizures in any patient. Finally, each patient became seizure-free after temporal lobectomy, which strongly supports a diagnosis of temporal lobe epilepsy.

According to Klass (18): "Most authors agree that EEG abnormalities are widespread, and probably involve diencephalic structures when consciousness is lost and when ictal automatism occurs." However, previous studies have documented occurrence of partial seizures without scalp ictal EEG discharges. In study of clinical and EEG manifestations of pentylenetetrazol-induced seizures (19), no changes in ictal EEG were reported in $18 \%$ of seizures. Devinsky et al. (20) reported that only $21 \%$ of SPS had an associated ictal EEG change. Other investigators have also confirmed that SPS and subclinical seizures usually do not have scalp EEG representation $(21,22)$. Walczak et al. (23) reported absence of scalp EEG ictal changes during 6 of 119 recorded CPS of temporal lobe origin. Whether these six clinical seizures were asso- ciated with EEG changes such as arrhythmic slowing or other changes from the baseline EEG of the type that did not constitute definite electrographic seizure activity is not clear.

Our descriptions of seizures that we consider psychogenically elaborated differ from other descriptions of temporal lobe seizures without scalp ictal EEG changes. We required that no scalp EEG changes of any kind occur during psychogenically elaborated events. In each case, we determined that the patient was amnesic for the event and manifested behavioral changes during psychogenically elaborated events that were atypical for the patient's habitual seizure. Finally, we also performed intracerebral and subdural recordings simultaneously with the scalp EEG recordings of these events.

Seizures that cause LOC can be classified as epileptic or nonepileptic by continuous scalp CCTVEEG monitoring, based in part on changes in scalp electrographic activity during epileptic events and absence of such a change during nonepileptic events. This tenet has widespread application in CCTV-EEG monitoring units for distinguishing between epileptic seizures and nonepileptic seizures when LOC has clearly been demonstrated in a spell (10). There may be exceptions to this rule, such as CPS arising from orbital frontal or mesial frontal cortex (described herein). Our observations generally support this tenet, but indicate that some events with LOC and no change in scalp EEG may include both epileptic and nonepileptic components. These atypical spells were semiologically distinct from each patient's habitual CPS. Care must be taken to record the patient's typical spells when a distinction between epileptic and nonepileptic seizures is sought in a laboratory setting $(10,24)$.

Acknowledgment: We thank Sally Mouilleseaux for assistance in manuscript preparation.

\section{REFERENCES}

1. Binnie CD, Rowan AJ, Overweg J, et al. Telemetric EEG and videomonitoring in epilepsy. Neurology 1981;31:298303.

2. Desai BT, Porter RJ, Penry JK. Psychogenic seizures: a study of 42 attacks in 6 patients, with intensive monitoring. Arch Neurol 1982;39:202-9.

3. Gumnit RJ. Behavior disorders related to epilepsy. In: Gotman J, Ives JR, Gloor P, eds. Long-term monitoring in epilepsy, Electroencephalogr Clin Neurophysiol Suppl 1985 35:313-23.

4. Holmes G, Sackellares JC, McKiernan J, Ragland M, Dreifuss FE. Evaluation of childhood pseudoseizures using EEG telemetry and video tape monitoring. I Pediatr 1980;97: 554-8.

5. King DW, Gallagher BB, Murvin M, et al. Pseudoseizuresdiagnostic evaluation. Neurology 1982;32:18-23.

6. Leis AA, Ross MA, Summers AK. Psychogenic seizures: 
ictal characteristics and diagnostic pitfalls. Neurology 1992; 42:95-9.

7. Luther JS, McNamara JO, Carville S, Miller P, Hope V. Pseudoepileptic seizures: methods and videoanalysis to aid diagnosis. Ann Neurol 1982;12:458-62.

8. Meierkord H, Will B, Fish D, Shorvon S. The clinical features and prognosis of pseudoseizures diagnosed using video-EEG telemetry. Neurology 1991;41:1643-6.

9. Ramani V. Intensive monitoring of psychogenic seizures, aggression and dyscontrol syndromes. In: Gumnit RJ, ed. Intensive neurodiagnostic monitoring. New York: Raven Press, 1987:203-17. (Advances in neurology, vol. 46.)

10. Rowan M, Gates JR, eds. Non-epileptic seizures. Boston: Butterworth-Heinemann, 1993.

11. Gumnit RJ, Gates JR. Psychogenic seizures. Epilepsia 1986; 27(suppl 2):S124-9.

12. Henry TR, Ross DA. Summary of epilepsy surgery protocols: the University of Michigan. In: Lüders HO, ed. Epilepsy surgery. New York: Raven Press, 1992;781.

13. Gloor P. Consciousness as a neurological concept in epileptology: a clinical review. Epilepsia 1986;27(suppl 2):S14-26.

14. Gloor P, Olivier A, Ives J. Loss of consciousness in temporal lobe seizures: observations obtained with stereotaxic depth electrode recordings and stimulations. In: Canger $\mathrm{R}$, Augelri F, Penry JK, eds. Advances in epileptology: XI Epilepsy International Symposium. New York: Raven Press, 1980:349-53.

15. Lothman EW, Bertram EH III, Stringer JL. Functional anatomy of hippocampal seizures. Prog Neurobiol 1991;37:1-82.

16. Quesny LF. Seizures of frontal lobe origin. In: Meldrum BS,
Pedley TA, eds. Recent advances in epilepsy, vol. 3. New York: Churchill Livingstone, 1986:81-111.

17. Williamson PD, Spencer DP, Spencer SS, Novelly RA, Mattson RM. Complex partial seizures of frontal lobe origin. Ann Neurol 1985;18:497-504.

18. Klass DW. Electroencephalographic manifestations of complex partial seizures. In: Penry JK, Daly DD, eds. Complex partial seizures and their treatment. New York: Raven Press 1975:113-40. (Advances in neurology, vol. 11.)

19. Ajmone-Marsan C, Ralston BL. The epileptic seizure. Its functional morphology and diagnostic significance. Electroclinical correlations. Springfield, IL: Charles C Thomas, 1957:139-76.

20. Devinsky O, Kelley K, Porter RJ, Theodore WH. Clinical and electroencephalographic features of simple partial seizures. Neurology 1988;38:1347-52.

21. Lesser RP, Lüders H, Dinner DS, Morris HH. Simple partial seizures. In: Lüders H, Lesser RP, eds. Epilepsy: electroclinical syndromes. London: Springer-Verlag, 1987:223-8.

22. Sperling MR, O'Connor MJ. Auras and subclinical seizures. Characteristics and prognostic significance. Ann Neurol 1990;28:320-8.

23. Walczak TS, Lewis DV, Radtke R. Scalp EEG differs in temporal and extratemporal complex partial seizures. $J$ Epilepsy 1991;4:25-8.

24. Sperling MR, Mendius RL. Postscript: strategies for semiinvasive and invasive approaches. In: Engel J Jr, ed. Surgical treatment of the epilepsies, 2nd ed. New York: Raven Press, 1993:451-4. 